\title{
Interval Estimation for the Exponential Distribution under Progressive Type-II Censored Step-Stress Accelerated Life-Testing Model Based on Fisher Information
}

\author{
Maryam Baghery and Fatemeh Yousefzadeh* \\ Birjand University
}

\begin{abstract}
This paper, determines the confidence interval using the Fisher information under progressive type-II censoring for the k-step exponential step-stress accelerated life testing. We study the performance of these confidence intervals. Finally an example is given to illustrate the proposed procedures.
\end{abstract}

Keywords. Accelerated life test; bootstrap confidence intervals; step-stress; exponential distribution; progressive type-II censoring; Fisher information; coverage percentage.

MSC 2010: 62F10.

\section{Introduction}

Products or materials are often tested at higher levels of stress than operational conditions to quickly obtain information on the life distribution or product performance under normal use. Such testing could save much time and money. In step-stress accelerated life test (SSALT), the stress for survival units is generally changed to a higher stress level at a pre-determined

* Corresponding author 
time. Determination of the stress change times is one of the most important design problems in SSALT. The exploit of modelling data from SSALT and developing inferences from such data have been studied by many authors. For example, Tang et al. (1999) discussed the MLEs for parameters in a multi-censored accelerated life tests. Some new models for SSALT had been proposed by Khamis and Higgins (1998). Tang and Yeo (2002) used the least square method to estimate the life-stress relationship in step-stress accelerated life test. Wang (2006) obtained unbiased estimations for the exponential distribution based on SSALT data. Point and interval estimation for the exponential simple step-stress model had been obtained by Balakrishnan et al. (2007).

This paper investigate the confidence interval for the exponential SSALT model under progressive type-II censoring based on Fisher information. Some basic assumptions in the exponential SSALT will be given in Section 2. Section 3 provides the expected Fisher information matrix $I_{i, j}(\theta)=E\left(-\frac{\partial^{2} \ln L(\alpha, \beta)}{\partial(\alpha, \beta)}\right)$. In Section 4 confidence Interval estimation (CIS) is performed by finding the percentile bootstrap. Section 5 gives a Monte Carlo simulation to study coverage percentages and discutient on the average interval lengths of estimators. In Section 6 an example is given to illustrate the proposed procedures. Finally, conclusions are included in Section 7.

\section{Basic Assumptions}

Under the exponential distribution, statistical inference in the SSALT is done by the following assumptions:

1. For any level of stress, the life of test units is exponentially distributed. At stress level $x_{i}$, it is given by:

$$
f_{i}(t)=\frac{1}{\theta_{i}} e^{-\frac{t}{\theta_{i}}} \quad t>0 .
$$

2. The mean life of $\theta_{i}$ of a test unit at stress level $x_{i}$, is a log-linear function of stress:

$$
\log \theta_{i}=\alpha+\beta x_{i}
$$

where $\alpha$ and $\beta$ are unknown model parameters.

3. A cumulative exposure model holds: The remaining life depends only on the current cumulative failure probability and current stress level regardless of how the probability is accumulated (Nelson, 1980). 
A SSALT model under progressive type-II censoring is as follows. Let all $n$ units of experiment are initially placed at lowest stress $x_{1}$. At the first failure time $t_{1,1}, R_{1,1}$ units are randomly removed from the remaining $n-1$ surviving units. At the second failure time $t_{1,2}, R_{1,2}$ units are randomly removed from the remaining $n-2-R_{1,1}$. The test continues until the $r_{1}$ th failure time $t_{1, r_{1}}$. At failure time $t_{1, R_{1}}, R_{1, r_{1}}$ units are randomly removed from the remaining $n-r_{1}-\sum_{j=1}^{r_{1}-1} R_{1, j}$ surviving units. Then the stress is changed to $x_{2}$. Similar to stress $x_{1}$, the test is continued until $r_{2}$ units have failed. At the stress $x_{2}, r_{2}$ failure times $t_{2, j}\left(j=1,2, \ldots, r_{2}\right)$ of test units are observed. At failure time $t_{2, j}, R_{2, j}$ units are randomly removed from the remaining $n-\sum_{i=1}^{r_{1}}\left(1+R_{1, i}\right)-j-\sum_{i=1}^{j-1} R_{2, i}$ surviving units. The stress is changed again, and the test continues until attaint $r_{k}$ th failure under the stress $x_{k}$. The failure times at the stress $x_{i}(i=1,2, \ldots, k)$ can be written as

$$
t_{i, 1} \leqslant t_{i, 2} \leqslant \ldots \leqslant t_{i, r_{i}} .
$$

Consider for $i=1,2, \ldots, k$ the lifetime at stress level $x_{i}$ has the exponential distribution under assumption 1, then the likelihood function is:

$$
L(\alpha, \beta) \propto \prod_{i=1}^{k} \theta_{i}^{-r_{i}} e^{\frac{-T_{i}}{\theta_{i}}},
$$

where

$$
\begin{aligned}
T_{i}= & \sum_{j=1}^{r_{i}}\left(1+R_{i, j}\right)\left(t_{i, j}-t_{i-1, r_{i}-1}\right) \\
& +\left\{n-\sum_{l=1}^{i}\left(r_{l}+\sum_{j=1}^{r_{l}} R_{l, j}\right)\right\}\left(t_{i, r_{i}}-t_{i-1, r_{i-1}}\right)
\end{aligned}
$$

\section{$3 \quad$ Fisher Information Matrix}

We have from Wang and yu (2009) that the MLE's of the parameters $\alpha$ and $\beta$ are given by:

$$
\frac{\partial \ln L(\alpha, \beta)}{\partial \beta}=\sum_{i=1}^{k} T_{i} x_{i} e^{-\beta x_{i}} \frac{\sum_{i=1}^{k} r_{i}}{\sum_{i=1}^{k} T_{i} e^{-\beta x_{i}}}-\sum_{i=1}^{k} r_{i} x_{i}=0
$$




$$
\frac{\partial \ln L(\alpha, \beta)}{\partial \alpha}=\sum_{i=1}^{k} T_{i} e^{-\alpha-\beta x_{i}}-\sum_{i=1}^{k} r_{i}=0
$$

In this section, we provide the expected Fisher information matrix for the progressively censored data. The Fisher information matrix $F$ is obtained by taking expectation of the negative of second partial derivatives of $\ln L(\alpha, \beta)$ with respect to $\alpha, \beta$. If we denote the $2 \times 2$ matrix $F$ by

$$
F(\alpha, \beta)=\left[\begin{array}{ll}
F_{1,1}(\alpha, \beta) & F_{1,2}(\alpha, \beta) \\
F_{2,1}(\alpha, \beta) & F_{2,2}(\alpha, \beta)
\end{array}\right]=-E\left[\begin{array}{cc}
\frac{\partial^{2} \operatorname{Ln} L(\alpha, \beta)}{\partial \alpha^{2}} & \frac{\partial^{2} \ln L(\alpha, \beta)}{\partial(\alpha, \beta)} \\
\frac{\partial^{2} \ln L(\alpha, \beta)}{\partial(\alpha, \beta)} & \frac{\partial^{2} \ln L(\alpha, \beta)}{\partial\left(\beta^{2}\right)}
\end{array}\right]
$$

The elements of this matrix are described as follow:

$$
\begin{gathered}
F_{1,1}(\alpha, \beta)=\sum_{i=1}^{k} \frac{1}{\theta_{i}} E\left(T_{i}\right)=\sum_{i=1}^{k} r_{i} \\
F_{2,2}(\alpha, \beta)=\sum_{i=1}^{k} \frac{x_{i}^{2}}{\theta_{i}} E\left(T_{i}\right)=\sum_{i=1}^{k} r_{i} x_{i}^{2} \\
F_{1,2}(\alpha, \beta)=F_{2,1}(\alpha, \beta)=\sum_{i=1}^{k} \frac{x_{i}}{\theta_{i}} E\left(T_{i}\right)=\sum_{i=1}^{k} r_{i} x_{i}
\end{gathered}
$$

where

$$
\begin{aligned}
E\left(T_{i}\right)= & \sum_{j=1}^{r_{i}}\left(1+R_{i, j}\right) E\left(t_{i, j}-t_{i-1, r_{i}-1}\right) \\
& +\left\{n-\sum_{l=1}^{i}\left(r_{l}+\sum_{j=1}^{r_{1}} R_{l, j}\right)\right\} E\left(t_{i, r_{i}}-t_{i-1, r_{i-1}}\right),
\end{aligned}
$$

which can be obtained by Wang and $\mathrm{Yu}(2009)$. The asymptotic variance covariance matrix of $\hat{\theta}=(\hat{\alpha}, \hat{\beta})$ can be obtained by inverting the Fisher information matrix.

\section{Bootstrap Confidence Intervals}

In this section we use the studentized-t bootstrap method (Hall, 1992) to construct CIS for the unknown parameters $(\alpha, \beta)$. We find the MLEs for the 
parameters $(\hat{\alpha}, \hat{\beta}, \hat{\theta})$ that depend only on the total test times $T_{1}, T_{2}, \ldots, T_{k}$ and the failure numbers $r_{1}, r_{2}, \ldots, r_{k}$ at stress levels $x_{1}, x_{2}, \ldots, x_{k}$, and not on the sample size $n$ and the removed numbers $R_{i, j}, i=1,2, \ldots, k, j=$ $1,2, \ldots, r_{i}$ at the failure times $t_{i, j}, i=1,2, \ldots, k, j=1,2, \ldots, r_{i}$. Further, note that $2 T_{i} / \theta_{i} \sim \chi^{2}\left(2 r_{i}\right)$, without loss of generality, we choose $R_{i, j}=0, i=$ $1,2, \ldots, k, j=1,2, \ldots, r_{i}$. Hence, in order to obtain the bootstrap sample of $(\hat{\alpha}, \hat{\beta}, \hat{\theta})$, we need only to consider various combinations $\left(r_{1}, r_{2}, \ldots, r_{k}\right)$. The bootstrap sample of $(\hat{\alpha}, \hat{\beta}, \hat{\theta})$ can be obtained by the following steps.

Step 1. From SSALT sample $t_{i, j}, i=1,2, \ldots, k, j=1,2, \ldots, r_{i}$, compute $(\hat{\alpha}, \hat{\beta}, \hat{\theta})$.

Step 2. Based on $(\hat{\alpha}, \hat{\beta})$ and $r_{1}, r_{2}, \ldots, r_{k}$ generate a random sample $\left(T_{1}^{*}, T_{2}^{*}\right.$, $\left.\ldots, T_{k}^{*}\right)$ of $\left(T_{1}, T_{2}, \ldots, T_{k}\right)$, where $\left(T_{1}, T_{2}, \ldots, T_{k}\right)$ are independent, and $2 T_{i} / \hat{\theta}_{i}$ $\sim \chi^{2}\left(2 r_{i}\right)$. Here $\hat{\theta}_{i}=\exp \left(\hat{\alpha}+\hat{\beta} x_{i}\right)$.

Step 3. Based on $\left(T_{1}^{*}, T_{2}^{*}, \ldots, T_{k}^{*}\right)$ compute the bootstrap sample estimates $\hat{\alpha}^{*}, \hat{\beta}^{*}, \hat{\theta}^{*}$ of $\alpha, \beta, \theta$, Then by inverting of Fisher information matrix, we obtain variances of the bootstrap sample estimates $\hat{\alpha}^{*}, \hat{\beta}^{*}, \hat{\theta}^{*}$.

Step4. Repeat steps 2-3 B times.

Then arrange all the values of $\hat{\alpha}^{*}, \hat{\beta}^{*}, \hat{\theta}^{*}$ in an ascending order to obtain the bootstrap samples. In any replication we have the statistics in the form of

$$
Z_{1}^{*,[i]}=\frac{\hat{\alpha}^{*,[i]}-\hat{\alpha}}{\sqrt{\operatorname{var}\left(\hat{\alpha}^{*}\right)}}, Z_{2}^{*,[i]}=\frac{\hat{\beta}^{*,[i]}-\hat{\beta}}{\sqrt{\operatorname{var}\left(\hat{\beta}^{*}\right)}}, \quad i=1, \ldots, B
$$

We now obtain the $\% 100(1-\gamma)$ confidence interval for $\alpha, \beta$ as

$$
\hat{\alpha}-Z_{1}^{*,\left(1-\frac{\gamma}{2}\right) B} \sqrt{\operatorname{var}(\hat{\alpha})}, \hat{\alpha}-Z_{1}^{*,\left(\frac{\gamma B}{2}\right)} \sqrt{\operatorname{var}(\hat{\alpha})}
$$

and

$$
\hat{\beta}-Z_{2}^{*,\left(1-\frac{\gamma}{2}\right) B} \sqrt{\operatorname{var}(\hat{\beta})}, \hat{\beta}-Z_{2}^{*,\left(\frac{\gamma B}{2}\right)} \sqrt{\operatorname{var}(\hat{\beta})}
$$

where $Z^{*,\left(1-\frac{\gamma}{2}\right) B}$ is the $\left(1-\frac{\gamma}{2}\right) B$ percentile of $Z^{*}$. We intend to present two confidence intervals, the bootstrap confidence and the exact confidence intervals that obtained from wang (2010). The results of simulation for two ways are reported in Tables 1 to 3 . 
36 Interval Estimation for the Exponential Distribution under Progressive Type-II ...

Table 1. Experiment schemes, coverage percentages and average interval lengthes in simulation study for $\alpha=4, \beta=-1$

\begin{tabular}{|c|c|c|c|c|c|c|}
\hline \multirow[b]{2}{*}{$\mathrm{k}$} & \multirow[b]{2}{*}{$\mathrm{r}$} & \multirow[b]{2}{*}{ Parameters } & \multicolumn{2}{|c|}{$\gamma=0.05$} & \multicolumn{2}{|c|}{$\gamma=0.1$} \\
\hline & & & Exact & Boot & Exact & Boot \\
\hline \multirow{4}{*}{2} & \multirow{4}{*}{$(9,6)$} & \multirow[t]{2}{*}{$\alpha$} & 0.9502 & 0.9497 & 0.8984 & 0.8954 \\
\hline & & & 5.2601 & 5.2560 & 4.3738 & 4.3843 \\
\hline & & \multirow[t]{2}{*}{$\beta$} & 0.9495 & 0.9457 & 0.8956 & 0.8945 \\
\hline & & & 8.6114 & 8.6099 & 7.1352 & 7.1766 \\
\hline \multirow{4}{*}{2} & \multirow{4}{*}{$(12,8)$} & \multirow[t]{2}{*}{$\alpha$} & 0.9507 & 0.9497 & 0.9008 & 0.8968 \\
\hline & & & 4.5280 & 4.5078 & 3.7707 & 3.7655 \\
\hline & & \multirow[t]{2}{*}{$\beta$} & 0.9493 & 0.9441 & 0.8968 & 0.8931 \\
\hline & & & 7.3912 & 7.3780 & 6.1489 & 6.1597 \\
\hline \multirow{4}{*}{2} & \multirow{4}{*}{$(15,10)$} & \multirow[t]{2}{*}{$\alpha$} & 0.9503 & 0.9481 & 0.8991 & 0.8953 \\
\hline & & & 4.0265 & 4.0100 & 3.5550 & 3.3525 \\
\hline & & \multirow[t]{2}{*}{$\beta$} & 0.9499 & 0.9468 & 0.8988 & 0.8971 \\
\hline & & & 6.5648 & 6.5587 & 5.4774 & 5.4829 \\
\hline \multirow{4}{*}{2} & \multirow{4}{*}{$(30,20)$} & \multirow[t]{2}{*}{$\alpha$} & 0.9522 & 0.9453 & 0.8975 & 0.8986 \\
\hline & & & 2.8136 & 2.8025 & 2.3468 & 2.3475 \\
\hline & & \multirow[t]{2}{*}{$\beta$} & 0.9504 & 0.9431 & 0.9004 & 0.8953 \\
\hline & & & 4.5849 & 4.5799 & 3.8299 & 3.8372 \\
\hline \multirow{4}{*}{3} & \multirow{4}{*}{$(12,8,6)$} & \multirow[t]{2}{*}{$\alpha$} & 0.9497 & 0.9498 & 0.8961 & 0.9014 \\
\hline & & & 2.8581 & 2.8572 & 2.3862 & 2.3865 \\
\hline & & \multirow[t]{2}{*}{$\beta$} & 0.9478 & 0.9505 & 0.8970 & 0.8997 \\
\hline & & & 3.9795 & 3.9815 & 3.3321 & 3.3217 \\
\hline \multirow{4}{*}{3} & \multirow{4}{*}{$(15,10,8)$} & \multirow[t]{2}{*}{$\alpha$} & 0.9460 & 0.9464 & 0.8932 & 0.8999 \\
\hline & & & 2.5064 & 2.5111 & 2.0990 & 2.0997 \\
\hline & & \multirow[t]{2}{*}{$\beta$} & 0.9467 & 0.9451 & 0.8948 & 0.9021 \\
\hline & & & 3.4689 & 3.4703 & 2.8961 & 2.8994 \\
\hline \multirow{4}{*}{3} & \multirow{4}{*}{$(30,20,15)$} & $\alpha$ & 0.9525 & 0.9482 & 0.9020 & 0.9016 \\
\hline & & & 1.7761 & 1.7739 & 1.4886 & 1.4853 \\
\hline & & $\beta$ & 0.9539 & 0.9458 & 0.9037 & 0.8997 \\
\hline & & & 2.4657 & 2.4658 & 2.0697 & 2.0640 \\
\hline
\end{tabular}


Table 2. Experiment schemes, coverage percentages and average interval lengthes in simulation study for $\alpha=4, \beta=-1$

\begin{tabular}{|c|c|c|c|c|c|c|}
\hline \multirow[b]{2}{*}{$\mathrm{k}$} & \multirow[b]{2}{*}{$\mathrm{r}$} & \multirow[b]{2}{*}{ Parameters } & \multicolumn{2}{|c|}{$\gamma=0.05$} & \multicolumn{2}{|c|}{$\gamma=0.1$} \\
\hline & & & Exact & Boot & Exact & Boot \\
\hline \multirow{4}{*}{3} & \multirow{4}{*}{$(60,40,25)$} & $\alpha$ & 0.9497 & 0.9469 & 0.9024 & 0.8949 \\
\hline & & & 1.2860 & 1.2868 & 1.0798 & 1.0786 \\
\hline & & $\beta$ & 0.9510 & 0.9457 & 0.9042 & 0.8934 \\
\hline & & & 1.8181 & 1.8214 & 1.5294 & 1.5269 \\
\hline \multirow{4}{*}{4} & \multirow{4}{*}{$(12,8,6,4)$} & $\alpha$ & 0.9543 & 0.9504 & 0.9061 & 0.8989 \\
\hline & & & 2.2715 & 2.2512 & 1.8967 & 1.8801 \\
\hline & & $\beta$ & 0.9525 & 0.9482 & 0.9046 & 0.8937 \\
\hline & & & 2.8176 & 2.7882 & 2.3519 & 2.3255 \\
\hline \multirow{4}{*}{4} & \multirow{4}{*}{$(15,10,8,6)$} & $\alpha$ & 0.9517 & 0.9465 & 0.9043 & 0.8987 \\
\hline & & & 1.9517 & 1.9482 & 1.6302 & 1.6300 \\
\hline & & $\beta$ & 0.9514 & 0.9491 & 0.9047 & 0.8993 \\
\hline & & & 2.3769 & 2.3603 & 1.9845 & 1.9731 \\
\hline \multirow{4}{*}{4} & \multirow{4}{*}{$(30,20,15,12)$} & $\alpha$ & 0.9512 & 0.9481 & 0.9031 & 0.9015 \\
\hline & & & 1.3667 & 1.3651 & 1.1432 & 1.1432 \\
\hline & & $\beta$ & 0.9514 & 0.9452 & 0.8992 & 0.8991 \\
\hline & & & 1.6659 & 1.6556 & 1.3915 & 1.3861 \\
\hline \multirow{4}{*}{4} & \multirow{4}{*}{$(60,40,25,18)$} & $\alpha$ & 0.9478 & 0.9492 & 0.8948 & 0.9011 \\
\hline & & & 1.0031 & 1.0020 & 0.8404 & 0.8406 \\
\hline & & $\beta$ & 0.9473 & 0.9478 & 0.8982 & 0.8975 \\
\hline & & & 1.2640 & 1.2603 & 1.0589 & 1.0569 \\
\hline \multirow{4}{*}{4} & \multirow{4}{*}{$(90,60,40,25)$} & $\alpha$ & 0.9516 & 0.9486 & 0.9008 & 0.8998 \\
\hline & & & 0.8256 & 0.8233 & 0.6910 & 0.6908 \\
\hline & & $\beta$ & 0.9494 & 0.9487 & 0.9016 & 0.8948 \\
\hline & & & 1.0386 & 1.0391 & 0.8716 & 0.8715 \\
\hline
\end{tabular}


38 Interval Estimation for the Exponential Distribution under Progressive Type-II ...

Table 3. Experiment schemes, coverage percentages and average interval lengthes in simulation study for $\alpha=1, \beta=1$.

\begin{tabular}{|c|c|c|c|c|c|c|c|c|}
\hline \multirow[b]{2}{*}{$\mathrm{k}$} & \multirow[b]{2}{*}{$\mathrm{r}$} & \multirow[b]{2}{*}{ Parameters } & \multicolumn{2}{|c|}{$\gamma=0.05$} & \multirow[b]{2}{*}{$\mathrm{k}$} & \multirow[b]{2}{*}{$\mathrm{r}$} & \multirow[b]{2}{*}{ Exact } & \multirow[b]{2}{*}{ Boot } \\
\hline & & & Exact & Boot & & & & \\
\hline \multirow{4}{*}{2} & \multirow{4}{*}{$(9,6)$} & $\alpha$ & 0.9510 & 0.9483 & \multirow{4}{*}{3} & \multirow{4}{*}{$(60,40,25)$} & 0.9500 & 0.9490 \\
\hline & & & 5.3864 & 5.2556 & & & 1.2257 & 1.22867 \\
\hline & & $\beta$ & 0.9500 & 0.9464 & & & 0.9500 & 0.9449 \\
\hline & & & 8.7053 & 8.6105 & & & 1.7863 & 1.8217 \\
\hline \multirow{4}{*}{2} & \multirow{4}{*}{$(12,8)$} & $\alpha$ & 0.9510 & 0.9496 & \multirow{4}{*}{4} & \multirow{4}{*}{$(12,8,6,4)$} & 0.9500 & 0.9511 \\
\hline & & & 4.5911 & 4.5041 & & & 2.2025 & 2.2513 \\
\hline & & $\beta$ & 0.9490 & 0.9473 & & & 0.9500 & 0.9447 \\
\hline & & & 7.2936 & 7.3742 & & & 2.8002 & 2.7883 \\
\hline \multirow{4}{*}{2} & \multirow{4}{*}{$(15,10)$} & $\alpha$ & 0.9500 & 0.9484 & \multirow{4}{*}{4} & \multirow{4}{*}{$(15,10,8,6)$} & .9490 & 0.9505 \\
\hline & & & 4.1258 & 4.0094 & & & 2.1293 & 1.9497 \\
\hline & & $\beta$ & 0.949 & 0.9447 & & & 0.9500 & 0.9483 \\
\hline & & & 6.721 & 6.5580 & & & 2.5141 & 2.3609 \\
\hline \multirow{4}{*}{2} & \multirow{4}{*}{$(30,20)$} & $\alpha$ & 0.9500 & 0.9451 & \multirow{4}{*}{4} & \multirow{4}{*}{$(30,20,15,12)$} & 0.9510 & 0.9483 \\
\hline & & & 2.7618 & 2.8016 & & & 1.2739 & 1.3643 \\
\hline & & $\beta$ & 0.9510 & 0.9454 & & & 0.951 & 0.9538 \\
\hline & & & 4.5671 & 4.5791 & & & 1.576 & 1.6553 \\
\hline \multirow{4}{*}{3} & \multirow{4}{*}{$(12,8,6)$} & $\alpha$ & 0.9500 & 0.9517 & \multirow{4}{*}{4} & \multirow{4}{*}{$(60,40,25,18)$} & 0.9490 & 0.9478 \\
\hline & & & 2.8950 & 2.8588 & & & 1.0050 & 1.0021 \\
\hline & & $\beta$ & 0.9500 & 0.9510 & & & 0.950 & 0.9447 \\
\hline & & & 4.0464 & 3.9821 & & & 1.251 & 1.2605 \\
\hline \multirow{4}{*}{3} & \multirow{4}{*}{$(15,10,8)$} & $\alpha$ & 0.9500 & 0.9509 & & & 0.95000 & 0.9491 \\
\hline & & & 2.6086 & 2.5118 & 4 & $(90,60,40,25)$ & 0.83818 & 0.8233 \\
\hline & & $\beta$ & 0.9500 & 0.9495 & & & 0.9500 & 0.9494 \\
\hline & & & 3.6377 & 3.4703 & & & 1.0386 & 1.0387 \\
\hline & & $\alpha$ & 0.9500 & 0.9503 & & & & \\
\hline 3 & $(30,20,15)$ & & 1.8884 & 1.7744 & & & & \\
\hline & & $\beta$ & 0.950 & 0.9493 & & & & \\
\hline & & & 2.668 & 2.4654 & & & & \\
\hline
\end{tabular}

\section{Simulation Study}

In this section we present the results of the Monte Carlo simulation study to compare exact and bootstrap confidence intervals. In each case, we have calculated the coverage percentages and average interval lengthes for different experiment schemes for the parameter $\alpha$ and $\beta$ at the stress levels $x_{0}=0.25, x_{1}=0.5, x_{2}=0.75, x_{3}=1, x_{4}=1.25$ and various combinations of the failure numbers $\left(r_{1}, \ldots, r_{k}\right)$. In this paper we chose the value of the parameters $\alpha=1,-1, \beta=4,1$. Note that the random variables 
$t_{i, j}$ for $i=1,2, \ldots, k$ and $j=1,2, \ldots, r_{i}$ follows the exponential distribution and we can compute the maximum likelihood estimators for progressive type-II censored, for the proposed confidence intervals. Note that $2 \frac{T_{i}}{\theta_{i}}$ follows the $\chi^{2}\left(2 r_{i}\right)$ distribution, we generate $k$ random numbers with the $\chi^{2}\left(2 r_{i}\right)(i=1,2, \ldots, k)$ distributions for each censoring scheme. We compute the proposed confidence intervals. Based on 10, 000 Monte Carlo simulations with $B=1000$ replications, the coverage percentages and average interval lengths of the proposed confidence intervals for the different $\left(r_{1}, r_{2}, \ldots, r_{k}\right)$ schemes are obtained. These values are tabulated in Tables 1 to 3 . We observe that by increasing the value of $k$ and $r$, for the parameters $\alpha$ and $\beta$ the coverage percentages is increased and average interval lengths is decreased. Based on the results of the simulation study, two methods provide a good balance between the coverage probabilities as well as average credible widths. However our recommendation for constructing confidence intervals is to apply the bootstrap method because its average credible widths is better than that of the exact method.

\section{Illustrative Example}

In this section we consider one example of section 4 of Wang and Fei (2003). This example is to obtain all the reliability indices of a kind of electronic components at the normal temperature of $x_{0}=25 \mathrm{C}$. Now units from a batch of products are randomly selected for the simple accelerated life model $\log \theta=\frac{1}{273.15+x}$. The accelerated temperature levels are $x_{1}=100 \mathrm{C}$ and $x_{2}=150^{\circ} C$. At $x_{1}=100^{\circ} C$, when 30 products have failed, the stress level rises to $x_{2}=150{ }^{\circ} \mathrm{C}$ and the test continues until 20 more products have failed. Their failure times are as follows. Failure times for the stress level $x_{1}=$ $32,54,59,86,117,123,213,267,268,273,299,311,321,333,339,386,408,422$, $435,437,476,518,570,632,666,697,796,854,858,910$. Failure times for the stress level $x_{2}=16,19,21,36,37,63,70,75,83,95,100,106,110,113,116,135$, $136,149,172,186$. These times are the differences $t_{2, j}-t_{1, r_{1}}$. This step-stress accelerated life testing is a SSALT with type-II censoring, which is a specific case about the progressive censoring scheme with $R_{i, j}=0, i=1,2, j=$ $1,2, \ldots, r_{i}$. For this example we obtained the $\% 95$ exact confidence intervals of $\alpha, \beta$ which are given by $[-8.6949,-0.9458],[3218.8,6246.4]$, respectively. The bootstrap confidence intervals for $\alpha, \beta$ are given by $[-9.9740,-0.4008]$, [2994.37, 6704.178], respectively. 


\section{Conclusions}

In this paper we discuss the type-II progressively censoring SSALT scheme. Assuming that the lifetime distributions are exponential distributed. We obtained the confidence intervals based on the bootstrap methods by fisher information, then access the coverage percentages and the average interval lengths for this parameters using assumptions.

\section{References}

Balakrishnan, N., Kundu, D., Ng, H.K.T. and Kannan, N. (2007). Point and interval estimation for a simple step-stress model with type-II censoring. J. Quality Technol. 39, 35-47.

Hall, P. (1992). On bootstrap confidence intervals in nonparametric regression. The Annals of Statistics, 20, 695-711.

Khamis, I. and Higgins, J.J. (1998). A new model for step-stress testing. IEEE Trans. Reliab., $R$ - 47, 131-134.

Nelson, W. (1980) . Accelerated life testing step-stress models and data analysis, IEEE Transactions on Reliability, 29, 103-108.

Tang, L.C., Goh, T.N., Sun, Y.S. and Ong, H.L. (1999). planning accelerated life tests for censored two-parameter exponential distributions. Naval Res. Logist. 46, 169-186.

Tang, S.L. and Yeo, K.P. (2002). A least-squares approach to analyzing life-stress relationship in step-stress accelerated life tests. IEEE Trans. Reliab. R-51, 177-182.

Wang, R.H. and Fei, H.L. (2003). Uniqueness of the maximum likelihood estimate of the Weibull distribution tampered failure rate. Commun. Stat. Theory Methods, 32, 2321-2338.

Wang, B.X. (2006). Unbiased estimations for the exponential distributions based on stepstress accelerated life-testing data. Appl. Math. Comput. 173, 1227-1237.

Wang, B.X. (2010). Interval estimation for exponential progressive type-II censored step-stress accelerated life-testing. Journal Of Statistical Planning and Inference. 140, 2706-2718.

Wang, B.X. and Yu, K. (2009). Optimum plan for step-stress model with progressive type-II censoring. Test. 18, 115-135. 
Maryam Baghery

Department of Statistics,

Birjand University,

Birjand, Iran.
Fatemeh Yousefzadeh

Department of Statistics,

Birjand University,

Birjand, Iran.

email: fyousefzadeh@birjand.ac.ir 